\title{
HgCdTe APDs detector developments for high speed, low photon number and large dynamic range photo-detection
}

Johan Rothman, E. De Borniol, S. Pes, A. Dumas, B. Hoareau, et al.

Johan Rothman, E. De Borniol, S. Pes, A. Dumas, B. Hoareau, S. Renet, L. Mathieu, J.-A. Nicolas, J.-P. Rostaing, J. le Perchec, G. Badano, P. Bleuet, P. Castelein, J. Abergel, S. Gout, X. Baudry, P. Ballet, J.-L. Santailler, "HgCdTe APDs detector developments for high speed, low photon number and large dynamic range photo-detection," Proc. SPIE 11852, International Conference on Space Optics - ICSO 2020, 118520F (11 June 2021); doi:

10.1117/12.2599159 SPIE. Event: International Conference on Space Optics - ICSO 2021, 2021, Online 


\section{International Conference on Space Optics-ICSO 2020}

Virtual Conference

30 March-2 April 2021

Edited by Bruno Cugny, Zoran Sodnik, and Nikos Karafolas
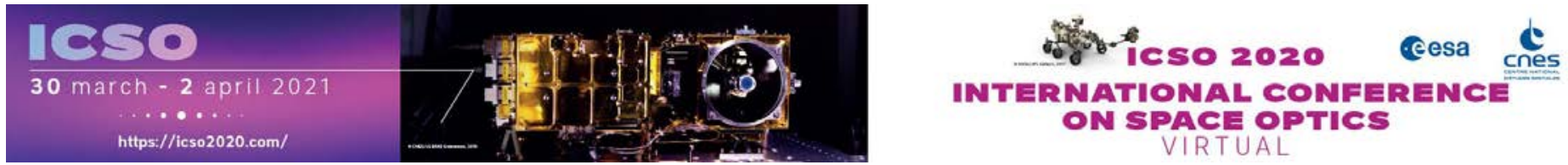

\section{HgCdTe APDs detector developments for high speed, low photon number and large dynamic range photo-detection}

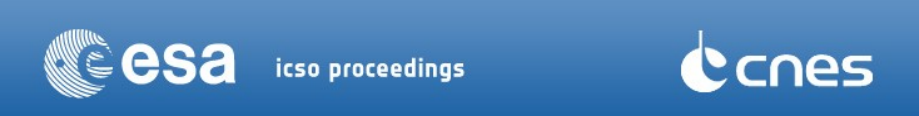




\title{
HgCdTe APDs detector developments for high speed, low photon number and large dynamic range photo-detection
}

\author{
J. Rothman ${ }^{\text {a }}$, E. De Borniol ${ }^{\text {a }}$, S. Pes, A. Dumas ${ }^{\mathrm{a}}$, B. Hoareau ${ }^{\mathrm{a}}$, S. Renet ${ }^{\mathrm{a}}$, L. Mathieu ${ }^{\mathrm{a}}$, J.-A. Nicolas ${ }^{\mathrm{a}}$, \\ J.-P. Rostaing, ${ }^{\mathrm{a}}$ J. le Perchec ${ }^{\mathrm{a}}$, G. Badano ${ }^{\mathrm{a}}$, P. Bleuet ${ }^{\mathrm{a}}$, P. Castelein ${ }^{\mathrm{a}}$, J. Abergel ${ }^{\mathrm{a}}$, S. Gout ${ }^{\mathrm{a}}$, X. \\ Baudry $^{\mathrm{a}}$, P. Ballet ${ }^{\mathrm{a}}$, J.-L. Santailler ${ }^{\mathrm{a}}$ \\ ${ }^{a}$ CEA/Leti Minatec Campus, 17, rue des Martyrs- 38054 Grenoble Cedex 9- France
}

\begin{abstract}
$\mathrm{HgCdTe}$ Avalanche Photo Diodes (APDs) are developed at CEA/Leti to enable applications that require the detection of information contained in a low number of photons in each spatial and/or temporal bin, such as LiDAR and free space optical communications. The requirements for such detectors are strongly application dependent, which is why both the $\mathrm{HgCdTe}$ APD technology and the proximity electronics, used to extract the detected photocurrent, needs to be optimized for each application. The present communication reports results obtained from the development of detectors for high dynamic range LiDAR applications, made within the scope of the H2020 project HOLDON, and high data rate FSO, made in collaboration with Mynaric Lasercom AG. For FSO applications, we have measured $10 \mathrm{GHz}$ bandwidth at unity gain for APDs with $10 \mu \mathrm{m}$ diameter. At higher APD gain and diameter, the BW is presently limited by carrier transit and by resistance-capacitance product in small and large area APDs, respectively. For LiDAR we have developed APDs with an made of an array of diodes in parallel with a diameter up to $200 \mu \mathrm{m}$ and large avalanche gain, $\mathrm{M}>100$, that will be hybridized with a dedicated CMOS amplifier. This circuit was designed to enable photon shot noise limited linear detection over a dynamic range of 6 order of magnitude of signal for observation times ranging from ns up to $\mu$ s. First characterizations made at unity APD gain shows that the HOLDON detector will meet most of the required performance parameters in terms of sensitivity and linear dynamic range.
\end{abstract}

Keywords: $\mathrm{HgCdTe}$, APD, free space optical communications, LiDAR, photon counting, bandwidth, dynamic range

\section{INTRODUCTION}

HgCdTe APDs have opened a new horizon in photon starved applications due to their exceptional performance in terms of high linear gain, low multiplication noise and high quantum efficiency. The high linear gain, in excess of 100, and low multiplication noise, characterized by an excess noise factor down to $\mathrm{F}=1.2-1.3$, are both due to an exclusive multiplication of electrons that have until presented been observed $\mathrm{HgCdTe}$ APD with a large range of geometries and Cd compositions. Both focal plane arrays (FPAs) and large single element detectors have been made using HgCdTe APDs and high performance devices are at present available to detect the information in photon fluxes with a low number of photon in each spatio-temporal bin at low loss [1-6]. At CEA/Leti, recent developments have been focused on single pixels with high temporal resolution with the goal to meet the requirements for emerging applications such as free space optical communications, in collaboration with ESA and Mynaric and atmospheric LiDAR, within the scope of the H2020 project HOLDON. The aim of the present communication is to present the status of the HgCdTe APD detector developments for high data rate FSO and for atmospheric LiDAR applications.

A common point of these developments is the need for a large optically active area of the detector to cope with the size and fluctuations in position of the light on the detector, due to variable and/or large field of view, tracking errors and wave front modulation within the aperture of the collecting telescope. The HgCdTe APD technology used at CEA/Leti was originally developed to meet the requirements for focal plane array application with small pixel pitch, optimized for small area APDs with diameters smaller than $10 \mu \mathrm{m}$. During the recent years, this original process has been adapted to form APDs with larger active junction diameter in order to be able to focus the light within the active area of the APDs or to reduce the number of elements in large area APDs formed using an array of detectors in parallel. The former is mandatory to optimize the bandwidth in the APD,s for high data rate FSO, and reduced latency for LiDAR applications. The latter could be in favor of an improved yield of functional APDs. The results from this development are reported in section 2. 
The detector development within HOLDON is made with the objective to meet the requirements for a large range of earth based, air-born or space-born atmospheric LiDAR applications. An application specific integrated circuit (ASIC) was designed within the project to meet the corresponding needs in terms of signal dynamic range and temporal resolution with the goal to preserve a photon shot-noise sensitivity over the full dynamic range, down to single photon sensitivity. The detector architecture, circuit functionalities and expected performance are reported in section 3.1. At present, the HOLDON ASIC has been hybridized and characterized with an $\mathrm{HgCdTe}$ photodiode with limited avalanche gain. This detector has enabled to validate most of the functionalities of the HOLDON ASIC although the ultimate sensitivity has not yet been demonstrated. The result of these first characterizations of the hybridized HOLDON detector module is reported in section 3.2 .

The obtained results and future perspective for the development of fast high dynamic range HgCdTe APDs detectors at $\mathrm{CEA} /$ Leti are discussed in the final section 5 .

\section{HGCDTE APD TECHNOLGY DEVELOPMENT}

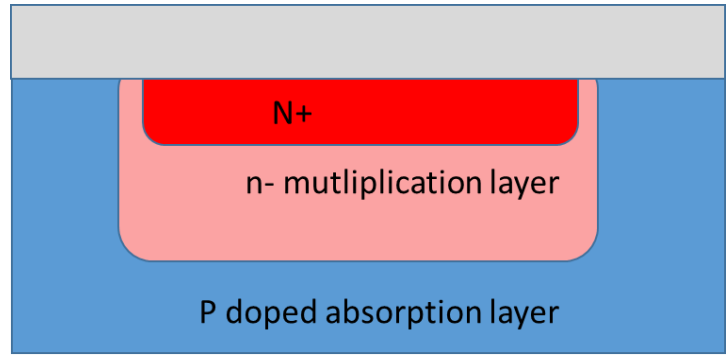

Figure 1. Schematic illustration of a $\mathrm{HgCdTe} \mathrm{APD}$ made at CEA/Leti with the traditional technology.

The HgCdTe APDs made at CEA/Leti are using a P type absorber, doped with intrinsic $\mathrm{Hg}$ vacancies, $\mathrm{VHg}$. The $\mathrm{VHg}$ level and the hole concentration in the absorption layer is typically in the range of $2-3 \times 10^{16} \mathrm{~cm}^{-3}$. An N on P type junction is formed by locally transforming the conductivity at the top surface of the absorbing layer to a highly doped N+ layer. This transformation is associated with the annihilation of $\mathrm{VHg}$ in proximity to the $\mathrm{N}+$ layer. This region is characterized by a low residual $\mathrm{N}$-type doping, with a residual donor concentration below $10^{15} \mathrm{~cm}^{-3}$. The corresponding $\mathrm{N}+\mathrm{n}-\mathrm{P}$ junction is illustrated in Figure 1. The electric field is concentrated within the n- layer in which the avalanche multiplication will take place. The performance of the resulting APD is strongly dependent on the geometry of the corresponding multiplication layer:

- The APD gain at a given reverse bias is directly related to the width of the multiplication layer. Wide multiplication layers will have lower gain due the need to apply a higher voltage to form an electric field in the junction that is higher than the critical field for the onset of the multiplication [7-8].

- The maximum useful APD gain is also dependent on the geometry and the width of the multiplication layer and the highest gain has so far been observed in APDs with wide multiplication layers, due to a relatively stronger reduction in tunnel current in such device [8-9].

- The bandwidth of the APDs is ultimately limited by the width of the multiplication layer, due to the transit time of the carriers generated during the avalanche process, in particular the slower holes generated at the end of the avalanche process [10]. On the other hand, the capacitance and, consequently, the de-charging time through the series resistance of the APD increases in narrow APDs and will limit the bandwidth in APDs with large diameter and/or with large series resistance.

- The excess noise factor has also been found to depend on the junction width. In [7], we reported on an increased value of $\mathrm{F}$ in wider APDs. The increased in $\mathrm{F}$ was attributed to an intrinsic mechanism that seemed to be reproduced using numerical modeling of the excess noise. The observed variations of $\mathrm{F}$ can also be attributed to spatial variation of the multiplication layer width that can introduce strong variations of the gain at different position of the APD. Recent founding, that will be discussed below, seem to indicate that this contribution can be of strong important, in particular for APDs with large diameters.

A main goal of the ongoing development of $\mathrm{HgCdTe}$ APDs is to form devices with a larger area of the multiplication layer. This is mandatory for optimizing the bandwidth as the charge collection must be made over a minimal distance and be 
accelerated by the presence of an electric field. A fast collection could also be of advantage for LiDAR application: such a collection should reduce the probability of the carrier to interact with trapping defects that could generate a slow low level response that could corrupt the data in some LiDAR scenarios.

The recent development of APDs with a diameter of $10 \mu \mathrm{m}$ and above have evidenced that the standard process used to form $\mathrm{HgCdTe}$ induced a strong asymmetry in the extension of the multiplication layer between the periphery and the center of the APD, as illustrated in Figure 1. This asymmetry leads to a limitation in the maximal useful APD gain, through the generation of strong tunnel currents at the junction periphery at low reverse bias. It will also introduce a strong variation in gain between the center and the periphery of the APD will introduced an extrinsic contribution to the excess noise factor. Such a variation of the gain is evidenced in Figure 2, that reports the spatial variation of the gain observed on one of the APDs made within the scope of the HOLDON project. The detector was made of an array of APD detectors at a pitch of $50 \mu \mathrm{m}$ with a diameter of $20 \mu \mathrm{m}$. At low revers bias (Figure 2a), the response is the highest in the center of APD and is slightly reduced when the light is injected between the APDs. At higher reverse bias and in presence of avalanche gain (Figure 2b), the response is found to be reduced in the center of each APD, indicating the presence of a reduced gain at this position. As the multiplication approaches the backside of the APD, this effect will be enhanced by the absorption of light in the multiplication layer that directly contributes to a reduced gain and increased multiplication noise.

If the central part of the junction extends through the full thickness of the absorption layer, there will be an even stronger detrimental effect on the performance. Under reverse bias, the electric field will not be able to establish through the central part of the junction, leading to an absence of gain. In addition, with a reduced central thickness of highly doped P-layer in the center of the APD will strongly impede the extraction of carrier in this region through an increase in series resistance, leading to a strongly increased response time.

The impact of the high asymmetry of the APD junctions was evidenced during the fabrication of APDs within the HOLDON project and during our efforts to optimize the BW of the APDs, in collaboration with Mynaric. Two batches of APDs were processed within the scope of the HOLDON project. In the first batch, the maximum APD gain was found to be limited by a strong contribution from peripheral tunnel current to values in the range of $M=20-30$. In the second batch, this tunnel current was strongly reduced using modified process parameters. A gain curve from an APD with a diameter of $150 \mu \mathrm{m}$, made from an array of 37 APDs at a pitch of $20 \mu \mathrm{m}$, is illustrated in Figure 3. A gain in excess of 1000 is observed without any signs of limiting tunnel or dark currents. However, response time measurement made with light focused on the backside of APDs from the same wafer have evidenced that the avalanche gain is very low in the center of the APD, associated with a slow response time. This correspond to an excessive extension of the central part of the junction and will result in a higher excess noise factor in these APDs. Consequently, APDs from both of the HOLDON batches will be used to form the HOLDON demonstrator detectors that will be delivered to the project partners for LiDAR demonstration. The APDs from the first batch will have a lower gain but a lower multiplication noise. The APDs from the second batch will have a higher gain, enabling down to single photon sensitivity, but at a higher multiplication noise and reduced information conservation.
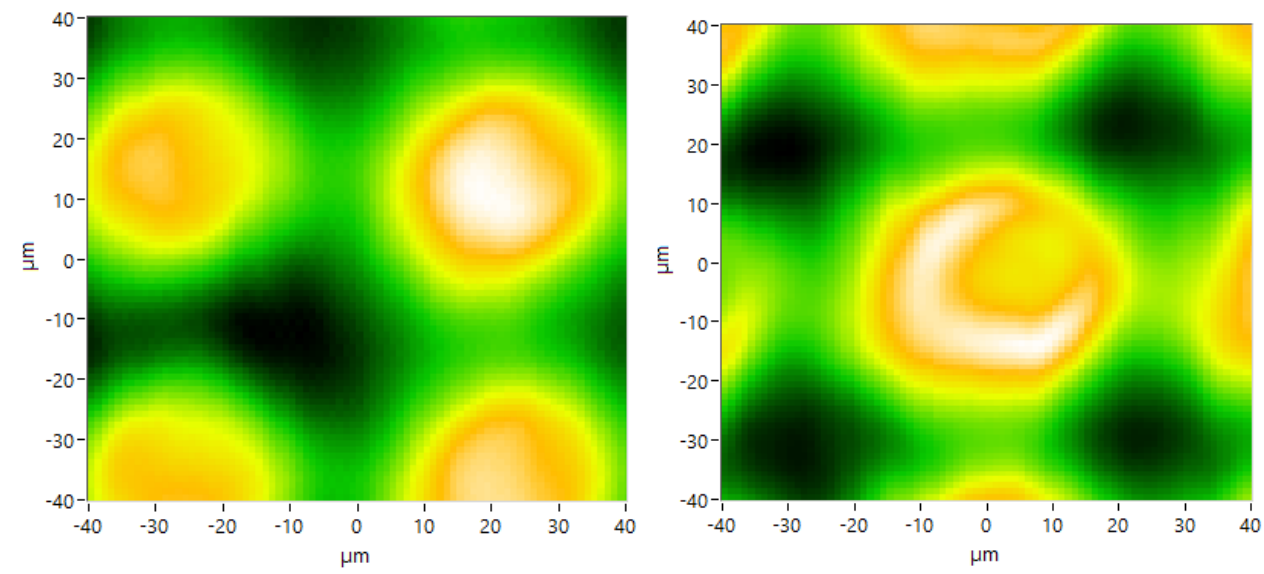

Figure 2. Photo-response map made on a HOLDON HgCdTe APD array with a $50 \mu \mathrm{m}$ pitch and $20 \mu \mathrm{m}$ APD diameter at 80 $\mathrm{K}$ and a) at low reverse bias of $0.5 \mathrm{~V}$ and $\mathrm{b}$ ) at high reverse bias of $5 \mathrm{~V}$ and with a developed APD gain. 


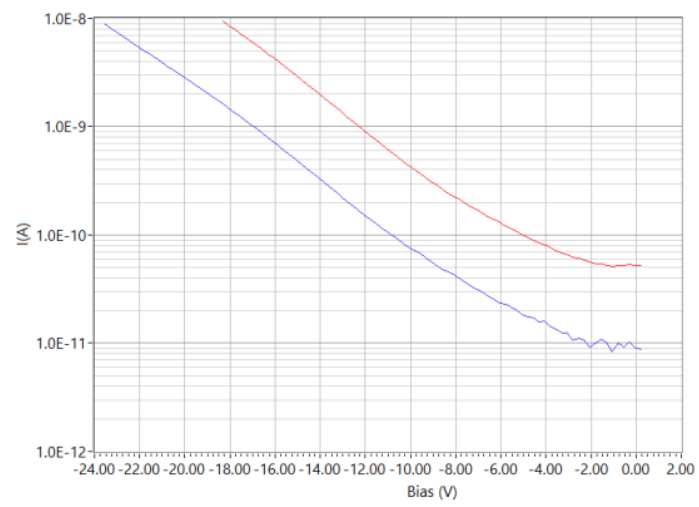

Figure 3. I(V) characteristics without and with additional flux measured at $80 \mathrm{~K}$ on a HOLDON HgCdTe APD array with APD pixels on a pitch of $20 \mu \mathrm{m}$ with an individual diameter of $8 \mu \mathrm{m}$, forming a macro-APD with a diameter of $150 \mu \mathrm{m}$. The parallelism of the two curves shows that the exponential increase is due to avalanche gain.

The development of $10 \mathrm{GHz}$ bandwidth APDs with avalanche gain in excess of 100 have also been delayed by the strong asymmetry of the multiplication layer in our old APD technology. Such a device requires to have a narrow central multiplication layer which will lead to a strong limitation avalanche gain due to the pinching of this layer at the periphery of the junction. A higher gain have been achieved in APD with larger central multiplication layer, associated with a high bandwidth at unity gain. The temporal response at an operating temperature of T=300 K of such an APD with a diameter of $10 \mu \mathrm{m}$ is reported in Figure 4. The unity gain impulse response time is characterized by a fall time of about $30 \mathrm{ps}$, corresponding to bandwidth close to $10 \mathrm{GHz}$. This is the first report of such high bandwidth in HgCdTe photodiodes. This results demonstrate that the carrier dynamics to collect and extract the charges in HgCdTe APDs are compatible with 10 $\mathrm{GHz}$ bandwidth even at room temperature. In this device, the response time increases with the gain at high reverse bias. This behavior is consistent with the contribution from avalanche-generated holes that needs to transit the wide multiplication layer, estimated to be in the range of $4 \mu \mathrm{m}$, to extract the all carriers from the APD [9-10]. The reported results is the first clear evidence of the influence of carrier multiplication and transit in the multiplication layer for an APD with exclusive electrons multiplication. The corresponding bandwidth at higher gain is about $3 \mathrm{GHz}$. A reduction of the central width of the multiplication layer by a factor 2-3 should enable a transit time limited bandwidth in the range of 6-9 $\mathrm{GHz}$, compatible with up to $12.5 \mathrm{Gbps}$ communications.

To achieve this ultimate goal, we are currently exploring a number of modified technological process' to form APDs with highly symmetric multiplication layers for a diameter up to $40 \mu \mathrm{m}$, associated with a BW in range of $10 \mathrm{GHz}$, a maximum avalanche gain in excess of 100 and an optimized multiplication noise. This development is still in progress and the corresponding results will be the subject of a future communication.

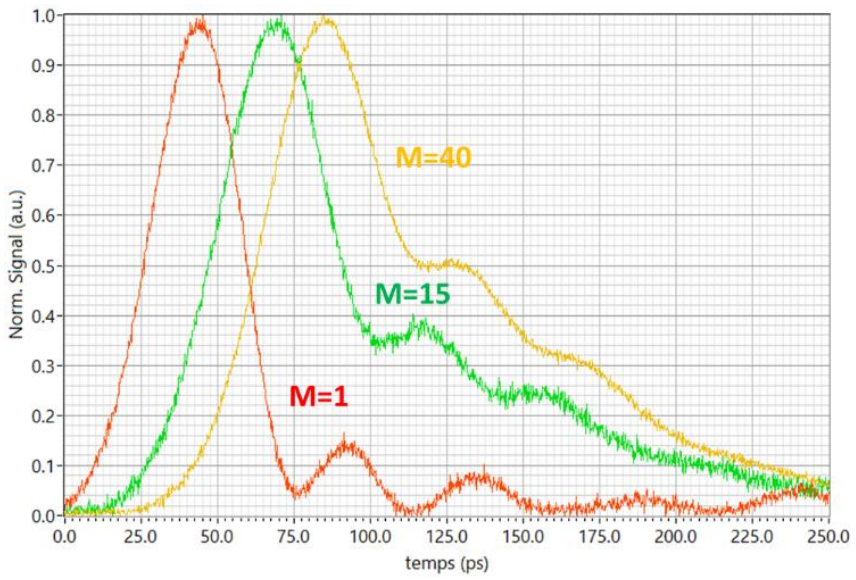

Figure 4. Impulse response measured at variable avalanche gain at $\mathrm{T}=300 \mathrm{~K}$ on a $10 \mu \mathrm{m}$ diameter APD with a $4 \mu \mathrm{m}$ wide multiplication layer. 


\section{HOLDON DETECTOR DEVELOPMENT}

The aim of the HOLDON H2020 project is to develop a versatile chain of detection, at CEA/Leti in collaboration with IDQuantique, that will be used by the other project partners (DLR, LMD, Airbus DS and Alter technologies) to demonstrate the use of the detector for a large range of atmospheric LiDAR applications. The long term goal is to dispose of a detector that meets the requirements for most space LiDAR application and that can be used "off the shelf" once it has been qualified for use in space.

At CEA/Leti we have worked on the optimization of the HgCdTe APD technology, as reported in the previous section and on the development of a new versatile ASIC that can be used at high performance over a large signal dynamic range and over variable observation times. The detector performance objectives have been defined with respect to the different LiDAR scenarios proposed by the partners are resumed in Table 1. In terms of $\mathrm{HgCdTe}$ performance, the goal of optimized performance can be expected for some of the APD geometries made within the first HOLDON batch but at lower gain. The higher gain APD from the second batch is expected to have a limited excess noise factor performance. The architecture and corresponding functionalities and expected performance of the HOLDON ASIC are reported in the section 3.1. The results from the first characterization of a HOLDON detector module made with an APD hybridized onto the HOLDON ASIC and integrated into a specially designed Liquide nitrogen (LN2) cryostat, are reported in the section 3.2.

Table 1. Expected characteristics of the HOLDON LiDAR detector module.

\begin{tabular}{|l|c|}
\hline \multicolumn{1}{|c|}{ Specification } & HOLDON Objective \\
\hline Excess noise factor F & 1.2 \\
\hline Quantum efficiency (QE) & $90 \%$ \\
\hline Quantum efficiency to excess noise Factor Ratio (QEFR) & $75 \%$ \\
\hline Bandwidth & $1 \times 10^{-5}$ \\
\hline Remanence at t > 5/BWAPD & 5 to $10000 \mathrm{~ns}$ \\
\hline Temporal resolution & $60 \mathrm{~dB}$ \\
\hline Photon Noise Limited Dynamic Range (PNDR) & $\left(10^{6}-10^{12} \mathrm{p} / \mathrm{s}\right)$ \\
\hline Spectral Range & 0.3 to $3 \mu \mathrm{m}$ \\
\hline Detector noise & $<1$ photon \\
\hline Minimum detected photon noise limited signal (photons/TC) & $<1$ photon \\
\hline Active Detector diameter & To be reduced using $\mu$ lenses \\
\hline
\end{tabular}




\subsection{HOLDON ASIC}

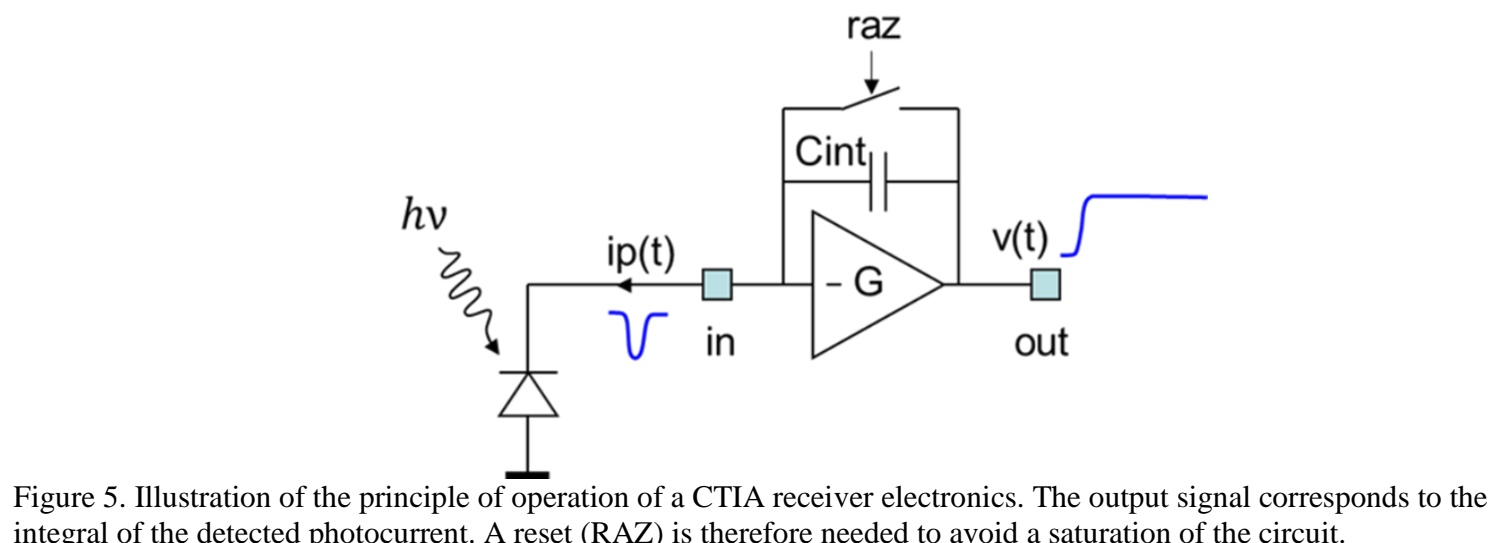

integral of the detected photocurrent. A reset (RAZ) is therefore needed to avoid a saturation of the circuit.

The main challenge for the design of the HOLDON ASIC was to find an architecture that enables to produce a highly linear detection that can handle more than 6 order of magnitudes in signal and at least 2 order in magnitudes in temporal resolution. The high dynamic range can only be achieved by implementing a variable gain amplifier. Two main configurations were considered: resistive and capacitive feed back trans-impedance amplifiers, RTIA and CTIA, respectively. An analysis of both configurations evidenced that CTIAs allow a more stable operation for a larger variation in feedback gain. In addition, the Johnson noise of the resistance in RTIAs gives an increased noise at higher gain values that limits the gain in sensitivity through the increase of gain. In a CTIA, a variation of the feedback capacitance will induce an increased reset noise that induces a constant signal offset that do not impact the temporal noise during the integration of the signal onto the capacitance.

Consequently, a multi-gain CTIA architecture was selected for the HOLDON ASIC. The operation of such an architecture is illustrated in Figure 5. At a difference to a RTIA, the output signal of the CTIA is proportional to the total amount of integrated charges since a reset of the feedback capacitance. The detection of a photon pulse is translated into a star-case signal proportional to the number of detected photons. The main drawback of this solution is that the charge integration on the capacitance can saturate in case of the detection of strong signals. This requires a good knowledge of the LiDAR scenario to adapt the gain and/or to insert a reset during the reception of the signal. The former can be dynamically changed during the observation by switching from a high to a lower gain. The latter will introduce a timing interval with a loss of data that need to be positioned in a region of expected low interest. This reset dead-time is expected to be lower than $50 \mathrm{~ns}$. To cope with the case of limited knowledge on the temporal variation of the LiDAR signal, we have also implemented an automatic reset function, that resets the capacity once a certain signal level have been obtained. This functionality allows avoiding a long dead time after saturation but might introduce reset dead-times at variable instants of time, that might introduce important loss of information.

The ASIC amplification chain is presented in Figure 6. The signal from the CTIA can be outputted from the ASIC using two modes of operation. The first, illustrated in Figure 6 a), is termed the continuous mode. In this mode, the signal is subject to an optional filtering before being directly amplified with a buffer that enable to charge the output with a impedance down to $50 \mathrm{Ohm}$. The output can be single ended with signal range of $2.4 \mathrm{~V}$, or differential, with a signal range of $4.8 \mathrm{~V}$. The latter can favorably be used to suppress common mode fluctuations and is directly compatible with differential input ADCs.

In the second mode, the signal is sampled in the ASIC. This mode is termed on chip sampling (OCS) and it is illustrated in Figure 6 b). In this mode, the signal is anlogly sampled on 2048 capacitors. The sampling is controlled by an external LVDS clock. The sampling frequency can be adapted to the expected information with asynchronous rate up to at least $100 \mathrm{M}$ samples/s. The recorded samples are sequentially read out at a slower rate. This enables to use an ADC with slower sampling rate associated with higher number of bits and larger dynamic range. The reduced bandwidth on the signal at the output on the buffer in the OCS mode do also allow to increase the charge at the input of the external sampling unit, that is expected to increase the linearity of the buffer amplifier. 
a)

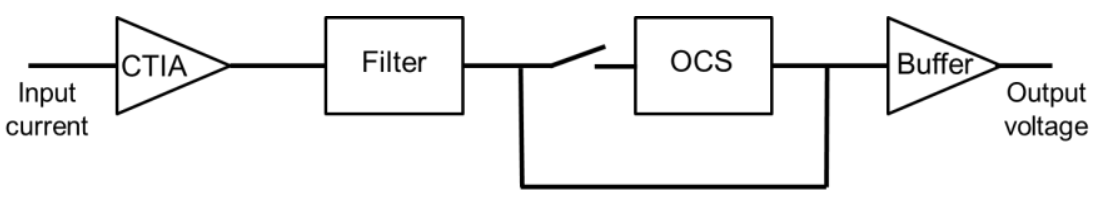

b)

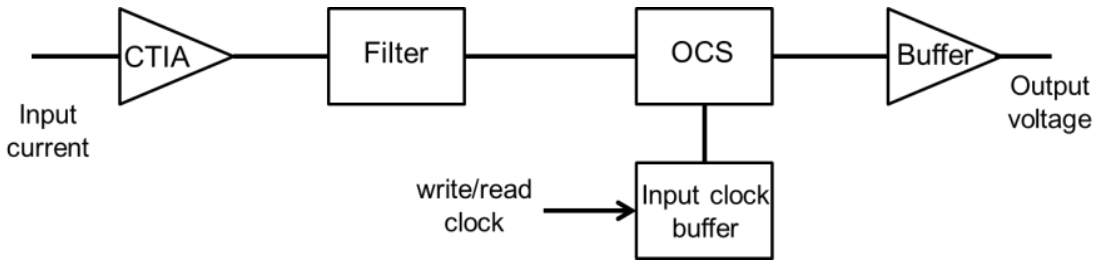

Figure 6. Illustration of the modes of operation of the HOLDON ASIC. In the continuous mode a), the output of the CTIA is directly accessible on the output of the ASIC. In the On Chip Sampling (OCS) mode, the signal is first analogy sampled at high rate, up to $100 \mathrm{MHz}$, within the ASIC and sequentially read at a lower rate, typically $5-10 \mathrm{MHz}$, in order to use a high resolution ADC with a high dynamic range and lower noise.

The choice of the CTIA architecture allowed us to implement 4 different gain values, each time varied by one order of magnitude, ranging from $\mathrm{C}_{\mathrm{G} 1}=10 \mathrm{fF}$ to $\mathrm{C}_{\mathrm{G} 4}=10 \mathrm{pF}$. Furthermore, the ASIC was made using the H9AROIC CMOS technology from STMircroelectronics. This process has a $130 \mathrm{~nm}$ resolution and thick oxide that sustain a maximum voltage of $4.8 \mathrm{~V}$. This high voltage is in favor of large linear dynamic range for each gain. Preliminary simulation has shown on a linear range of $2.4 \mathrm{~V}$ for each gain setting. The Table 2 resumes the expected performance in terms of signal range, noise and linearity for the different CTIA gains. A very low noise is obtained at all gains defining a dynamic range, up to $92 \mathrm{~dB}$ for the two highest gains, that is difficult to conserve using commercial ADC with sampling rates in excess of $100 \mathrm{MSamples} / \mathrm{s}$. It is strained that this high dynamic range is combined with a down to single photon level detection when combined with and APD gain in excess of 100 at the two highest gain settings G1 and G2.

Table 2. Expected characteristics of the versatile HOLDON ASIC for LiDAR applications.

\begin{tabular}{|c|c|c|c|c|c|c|c|c|c|}
\hline Gain & $C(f F)$ & $\begin{array}{c}\text { Signal } \\
\text { range }(\mathrm{V})\end{array}$ & $\begin{array}{c}\text { Charge capacity } \\
\text { (Melectrons) }\end{array}$ & Noise $(\mu \mathrm{V})$ & $\begin{array}{c}\text { Electron } \\
\text { noise rms } \\
\mathrm{M}=1\end{array}$ & $\begin{array}{c}\text { Electron } \\
\text { noise rms } \\
\mathrm{M}=100\end{array}$ & $\begin{array}{l}\text { Dynamic } \\
\text { range }(\mathrm{dB})\end{array}$ & \begin{tabular}{|c|} 
Full range \\
linearity \\
fluctuations \\
$(\%)$
\end{tabular} & $\mathrm{BW}(\mathrm{MHz})$ \\
\hline G1 & 10 & 2.4 & 0.15 & 390 & 24.3 & 0.2 & 75.8 & 2 & 3 \\
\hline
\end{tabular}

The expected linearity at each gain is strongly dependent on the gain of the CTIA, mainly through the variations of parasitic capacitances that have a strong voltage dependence. This result shows that the APD gain is not only useful to reduce the impact of the amplifier noise, it do also increase the linearity of the amplifier as it allows to integrate a small signal on a larger capacitance. For example, the G1 at unity APD gain and the G4 at a gain of 100 are characterized by a similar input noise, but the linearity has been improved by 2 order of magnitudes using the APD gain. The feature could be of high importance for the most demanding LiDAR applications.

As mentioned above, the internal high dynamic range of the HOLDON ASIC is difficult to conserve using presently available commercial ADCs. This problem has been solved by the implementation of the OCS mode that allows to use a higher performance slower ADC with higher dynamic range. The benefit in terms of dynamic range (maximum signal to noise ratio) for the HOLDON detection chain in which the signal is sampled in a proximity electronics made by IDQuantique, is reported in Table 3. It can be seen that the reduced noise and increased signal gives an increase of $14 \mathrm{~dB}$ in dynamic range for this mode. The difference can be reduced by applying numerical filters on the signal sampled with the continuous mode. In addition, the use of a higher input impedance at the input of the ADC for the OCS mode is expected to result in a higher linearity for this mode, due to a reduced output current from the buffer amplifier. 


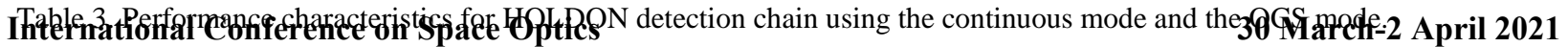

\begin{tabular}{|c|c|c|c|c|c|c|c|c|c|c|c|c|c|}
\hline \multirow[b]{2}{*}{ Mode } & \multicolumn{2}{|c|}{ HOLDON ASIC } & \multicolumn{4}{|c|}{ Input buffer amplifier } & \multicolumn{4}{|c|}{$A D C$} & \multicolumn{3}{|c|}{ HOLDON detection chain } \\
\hline & \begin{tabular}{|l|} 
Differential \\
voltage pp
\end{tabular} & $\begin{array}{l}\text { Differential } \\
\text { noise }(\mu \mathrm{V}) *\end{array}$ & $\begin{array}{c}\text { Differential buffer } \\
\text { amplifier }\end{array}$ & $\begin{array}{c}\text { Input charge } \\
\text { (kOhm) }\end{array}$ & $\mathrm{BW}(\mathrm{MHz})$ & Noise $(\mu \mathrm{V})$ & $A D C$ & $\begin{array}{l}\text { Differential } \\
\text { Voltage pp }\end{array}$ & ADC nosie $(\mu \mathrm{V})$ & $\begin{array}{c}\text { dynamic range } \\
\text { (dB) }\end{array}$ & \begin{tabular}{c|} 
Differential \\
voltage pp
\end{tabular} & $\begin{array}{c}\text { Total noise } \\
(\mu \mathrm{V})\end{array}$ & $\begin{array}{l}\text { Dynamic } \\
\text { range }\end{array}$ \\
\hline Continous & 3.8 & $60-390$ & LTC6419 & 0.1 & 300 & 56 & ADS54J69 & 1.9 & 379.10 & 74 & 1.9 & 387.9 & 73.8 \\
\hline OCS & 4.8 & $120-780$ & LTC6419 & 1 & 20 & 41.9 & LTC2387-18 & 8 & 142.26 & 95 & 4.8 & 190.8 & 88.0 \\
\hline
\end{tabular}

\subsection{First characterization of a HOLDON detector module}

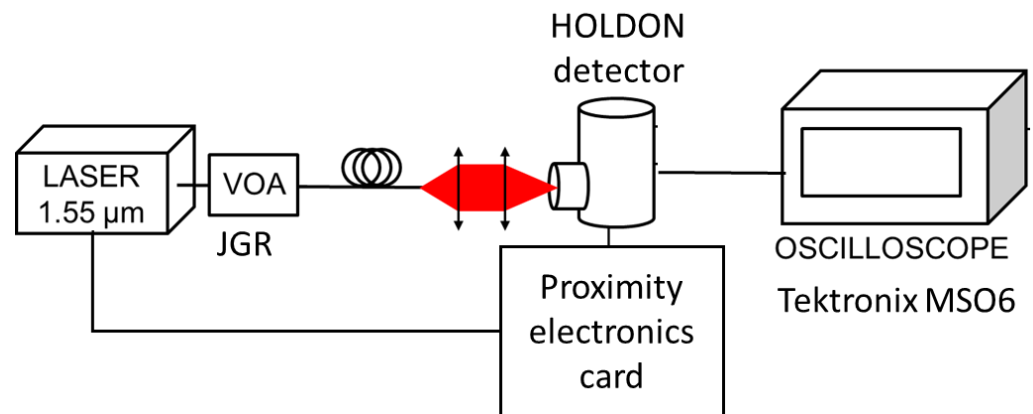

Figure 7. Experimental set-up used for the characterization of the first HOLDON detector module.

First characterization of a HOLDON detector module was made with an HOLDON ASIC hybridized with an $80 \mu \mathrm{m}$ diameter APD (array of $4 \mathrm{x} 4$ pixels at a $20 \mu \mathrm{m}$ pitch). This APD was extracted from the edge of the wafer and did not allow to apply a high reverse bias. Consequently, this detector module could not be characterized at high APD gain, which is why the characterization presented here has only been made close to unity gain with the objective to validate the different functionalities of the HOLDON ROIC. The measurement set-up is illustrated in Figure 7. At present, the HOLDON ASIC was controlled using a dedicated proximity electronics card that was developed at CEA/Leti and generic control electronics used to control focal plane arrays. The signal was sampled using a 12 bit Tektronix MSO6 oscilloscope. This instrument allows to correctly estimate the noise of the different operation modes, but it cannot be used to sample a signals over the full dynamic range of the HOLDON detector. The signal onto the detector was generated using a pulsed Laser from CALMAR optcom at wavelength of $\lambda=1.55 \mu \mathrm{m}$. The pulse energy and average laser power was controlled with Variable Optical Attenuator (VOA) from JGR with an attenuation range of $100 \mathrm{~dB}$ with an accuracy of $0.1 \mathrm{~dB}(2.4 \%)$.

At present, all detector module functionalities have been qualitatively validated.

Continuous mode operation: The continuous mode operation is functional with a $100 \mathrm{Ohm}$ differential charge or single ended $50 \mathrm{Ohm}$ charge on one of the outputs. Figure 8 reports signals recorded for the four different CTIA with variable input signal levels. The variation in CTIA gain between each setting was examined by measuring the variation of accumulated signal for 16 consecutive pulses at different signal power. The resulting signal variation for the different gains is reported in Figure 9. It can be seen that the gain slope is shifted by one order of magnitude between the two lowest gains, G3 and G4. The variation between the high gains, G1 and G2, is slightly reduced. This indicates that the feed-back capacitance is slightly higher in these cases. The impact of the CTIA gain on the BW is clearly visible on the modification of the rise time observed for the different gains. A minimal rise time of $9 \mathrm{~ns}$ is observed at the lowest gain. This is slightly longer than the HOLDON requirements of $5 \mathrm{~ns}$ and to the expected ASIC bandwidth of $300 \mathrm{MHz}$. This slower response can be explained by the response time of the APD, dominated by the diffusion within the multi element APD. It can also be due to a limitation of the bandwidth of the CTIA due to a high total input capacitance. The influence of the input capacitance is partially confirmed for the two highest gains, for which the corresponding bandwidths, 22 and $1.8 \mathrm{MHz}$, are slightly lower than the expected values of 30 and $3 \mathrm{MHz}$, for G2 and G1, respectively. The noise level for the different gains were measured to be close to the expected performance reported in Table 2. These results shows that, in combination with a high APD gain, the HOLDON detector will be able to reach single photon detection in combination with a very large linear dynamic range. 


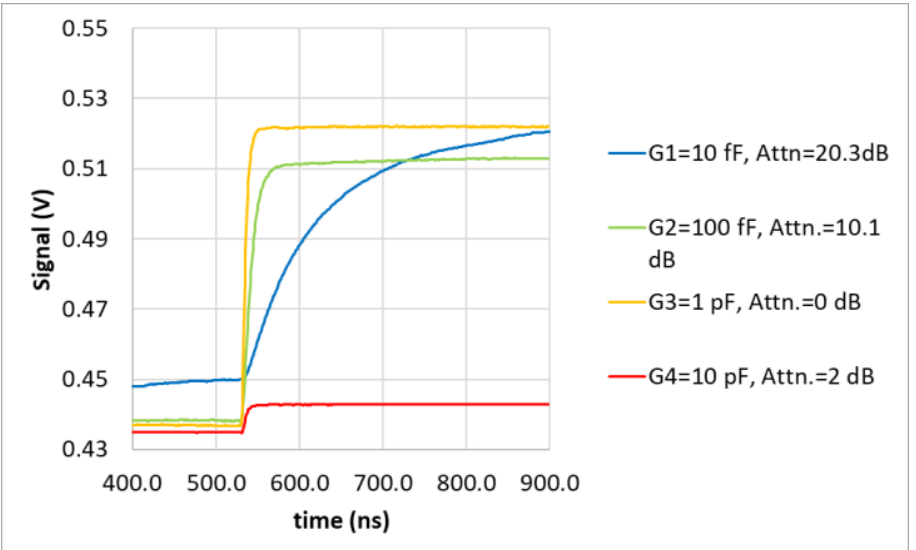

Figure 8. Impulse response measured on with a HOLDON detector module at unity gain using the four different gains and with variable attenuation of the laser power.

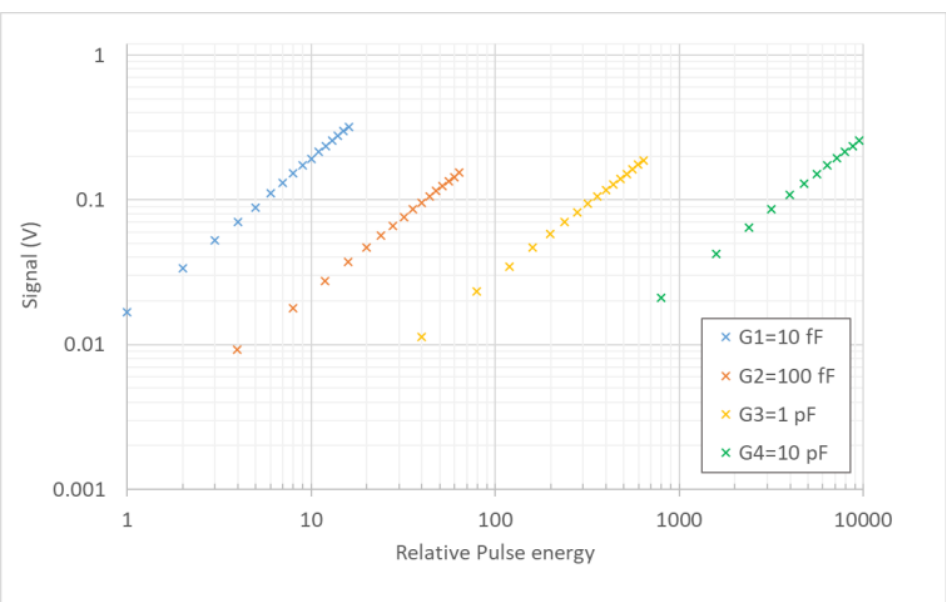

Figure 9. Detector linear response measured in the continuous mode for the different gains using a single ended signal with $1 \mathrm{MOhm}$ charge on the oscilloscope.

OCS mode operation: the OCS mode has been validated for all the 2048 on chip samples at sampling rates up to 100 Msamples/s. The operation has been validated with a charge up to $1 \mathrm{MOhm}$ on each output, which shows that a 1000 $\mathrm{kOhm}$ differential charge on the input of the IDQ electronics is compatible with the ASIC output signal for the OCS mode. As discussed above, this should be in favor of a higher dynamic range for this mode of operation.

Variable CTIA gain: a gain variation slightly less than 3 orders of magnitudes for the four gain settings has been confirmed as can be seen in Figure 9. We have also validated that the gain can be switched dynamically from a large gain to smaller gain without reseting the detector. This procedure can be used in LiDAR in which a smaller signal, for example from scattering in the atmosphere, is detected before a stronger signal, such as a ground echo. Switching from a smaller gain to a larger gain is also possible but requires a reset that will introduce a signal dead-time.

Single ended output voltage range: At high load impedance, the detector can output signals variations in excess of 2.4 $\mathrm{V}$ with an apparent linear behavior.

Differential output voltage range: The differential output is generated by forming a inverted copy of the CTIA output signal $\mathrm{V}_{\mathrm{SP}}$, around a common mode voltage $\mathrm{V}_{\mathrm{DA}}: \mathrm{V}_{\text {signal }}=2 \mathrm{~V}_{\mathrm{DA}}-\mathrm{V}_{\mathrm{SP}}$. For a signal that starts at a level $\mathrm{V}_{0}$ the total dynamic range is $2 \mathrm{~V}_{D A}-2 \mathrm{~V}_{0}$. $\mathrm{V}_{\mathrm{DA}}$ and $\mathrm{V}_{0}$ are generated internally and the observed values are close to the expected values, $2.4 \mathrm{~V}$ and $1.2 \mathrm{~V}$, respectively, corresponding to a differential signal output range of $4.8 \mathrm{~V}$. Both values can be modified by externally control voltages to increase or reduce the range. This functionality has been validated during the present tests. 
Linear dynamic range: The linear dynamic range has been characterized using the continuous mode with a single ended signal charged to $50 \mathrm{Ohm}$. In this mode, the signal amplitude and noise is divided by a factor between 2-3 due to the output impedance of the ASIC and the addition of additional resistance within the cryostat during the present measurements. A pulsed laser signal with an amplitude that was varied over more than 4 orders of magnitude was used to generate an impulse response. The variation of the detected signal as a function of the input signal is reported in Figure 10 measured at a CTIA gain G2=100 fF. The amplitude was estimated after an average of 200 waveforms to reduce the impact of noise, in particular from temporal fluctuations of the laser intensity. It can be seen that the detector is displaying a linear response over the explored dynamic range. The deviations from a linear response are reported in Figure $10 \mathrm{~b}$ ). A fluctuation of about $+/-2.5 \%$ is observed for all but the extreme measurements. This variation close to the expected precision of the VOA. At the lowest signal level, the larger deviation can be explained by the residual noise on the estimation of the very low amplitude, that was lower than the detector noise. The deviation at the largest signal level, could possibly be related to the use of the VOA at $0 \mathrm{~dB}$ attenuation that can be subject to a larger error. The maximum amplitude was $0.7 \mathrm{~V}$, corresponding to about $2 \mathrm{~V}$ at the output of the ASIC. As the upper and lower signal levels can be extended by more than two order of magnitudes by increasing (two order of magnitude) and decreasing (one order of magnitude) the CTIA gain, the results indicate that the linear dynamic range of the HOLDON detector exceeds the expected 6 order of magnitude of dynamic range. This conclusion do still need to be confirmed with a fully functional detector module at high APD gain.

Reset dead time: The external reset of the feedback capacitance is controlled by a $1.8 \mathrm{~V}$ logic signal. At present, the dead time have been observed to be in the order of $50 \mathrm{~ns}$, which is consistent with the expected value.

Auto reset: The auto-rest have been found to be fully operational.

Internal switchable filters : The four different filter settings have been validated, enabling internal filtering at 3, 30 et $300 \mathrm{MHz}$. This functionality is particularly interesting for the OCS mode to minimize the sampling of high-frequency noise.
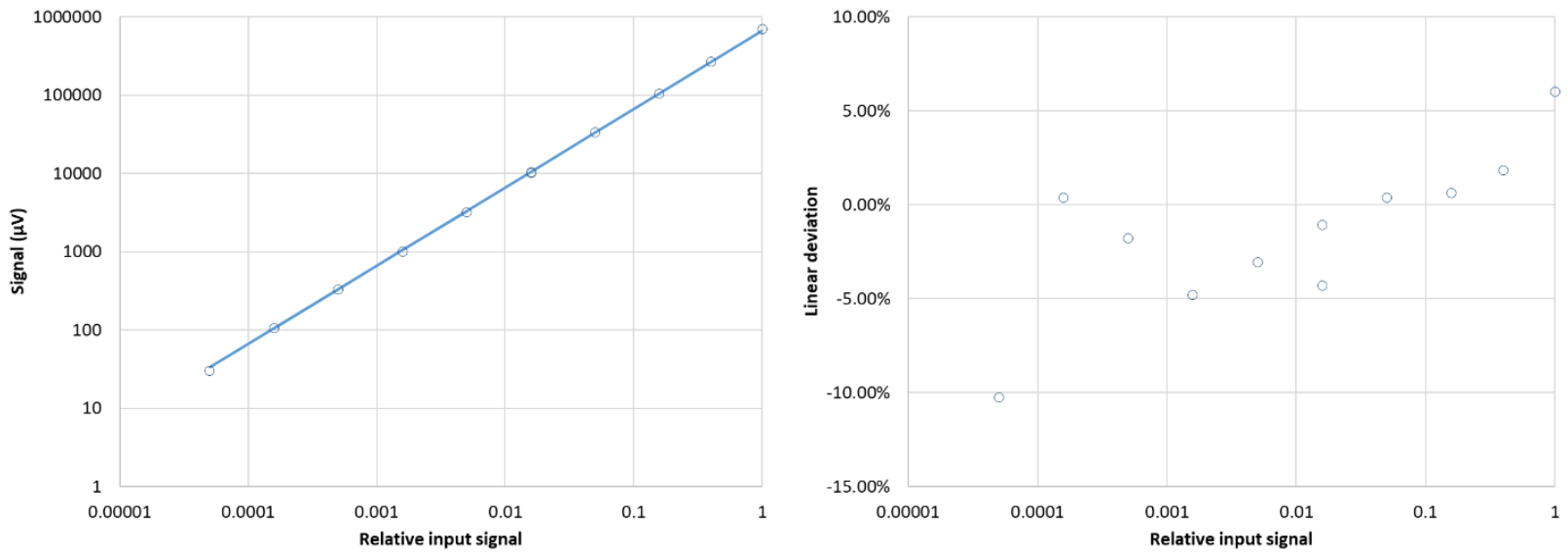

Figure 10. Detector linear response measured in the continuous mode with a gain $\mathrm{G} 2=100 \mathrm{fF}$ using a single ended signal with $50 \mathrm{Ohm}$ charge on the oscilloscope.

\section{CONCLUSION AND PERSPECTIVES}

$\mathrm{HgCdTe}$ APD detectors for time resolved and large dynamic range applications such as FSO and LiDAR are developed at CEA/Leti. Both applications are pushing for the development of APDs with diameters larger than $10 \mu \mathrm{m}$. The standard technology used at CEA/Leti for HgCdTe APDs has been found to form APDs with a high asymmetry between the central and peripheral part of the multiplication layer. This asymmetry yields a strong limitation in maximal APD gain, due the generation of tunnel current, and to the maximal bandwidth that can be reached at gain. The latter follows from the need of having narrow central multiplication width that will lead to an even stronger peripheral tunnel current and lower useful APD gain. In addition, the asymmetry will lead to a variation in gain between the center and periphery of the APD, leading an increase in the excess noise factor $F$. Pushing this technology to its limits in large diameter APDs, we have shown that large gains, in excess of 100, can be achieved but at the expense of low central gain. A bandwidth of $10 \mathrm{GHz}$ was observed at unity gain in an APD with a diameter of $10 \mu \mathrm{m}$ and a large multiplication layer of about $4 \mu \mathrm{m}$ at $300 \mathrm{~K}$. This result showed the carrier collection and extraction in $\mathrm{HgCdTe}$ APDs is compatible with $10 \mathrm{GHz}$ operation at $300 \mathrm{~K}$. The carrier 
transit in the wide multiplication layer did however limit the bandwidth at APD gain up to $M=40$. Present developments are dedicated to the optimization of the APD technology to form APDs with more symmetric multiplication layer with higher useful gain, higher bandwidth and lower excess noise.

The detector module development for LiDAR applications is developed within the scope of the H20202 project HOLDON. The aim is to develop a versatile detector that can meet the requirement for a large range of atmospheric LiDAR applications. A CMOS ASIC has been developed to meet these requirements when hybridized to a large area $\mathrm{HgCdTe}$ APD. A CTIA architecture was selected to design an amplifier with a linear gain over more than 6 orders of magnitude of the signal. In this architecture, the APD gain is not only useful to reduced the impact of the amplifier noise, it do also enable to use a larger feed back capacitor associated with a higher linearity. The high dynamic range was obtained by implementing a variable gain, over 3 order of magnitude, with a large dynamic range for each gain. An on chip sampling (OCS) mode was implemented in order to optimize the dynamic range at each gain. The OCS allows a fast analog sampling of the signal in the ASIC, associated with a slow readout that allows to use a slower ADC with larger dynamic range and lower noise. The resulting in a dynamic range is $88 \mathrm{~dB}$ for this mode at the lowest CTIA gains. First tests of the HOLDON ASIC hybridized with an HgCdTe APD has allowed to validate most of the detector functionalities. In particular, both the continuous mode and the OCS mode were found to be operational with noise levels close to the expected values. The high linear dynamic range was validated from measurements with variable CTIA gain, that can be varied by 3 orders of magnitude, and from linearity measurement with variable pulse amplitude at constant CTIA gain. The latter showed on a linear response over more than 4 orders of magnitude. The combined linear dynamic range exceeds the expected 6 orders of magnitude. The only characteristics that has not yet been confirmed is the response time, that was limited to a rise time of $9 \mathrm{~ns}$ at the lowest gain. This limitation is likely to be due to the response time of the APD.

In the short-term perspective, we will characterize new detector hybrids with high gain APDs using the full chain of detection developed within HOLDON, including a dedicated control electronics developed by IDQ. The detectors will then be delivered to the project partners for test in different LiDAR applications.

In a long-term perspective, both the high bandwidth optical communication application and the HOLDON detector will benefit from our ongoing development of the APD technology in favor of a larger gain, lower excess noise and higher bandwidth.

\section{ACKNOWLEDGEMENTS}

This work has been made with the support from CNES, from the European Union's Horizon 2020 research and innovation programme under grant agreement no 776390and Mynaric Lasercom GmbH.

\section{REFERENCES}

[1] Rothman, J., Lasfargues, G., Delacourt, B., Dumas, A., Gibert, F., Bardoux, A., Boutillier, M., "HgCdTe APDs for time-resolved space applications", CEAS Space J. 9, 507 (2017)

[2] A. Dumas, J. Rothman, F. Gibert, D. Édouart, G. Lasfargues, C. Cénac, F. Le Mounier, J. Pellegrino, J.-P. Zanatta, A. Bardoux, F. Tinto, and P. Flamant, "Evaluation of a HgCdTe e-APD based detector for $2 \mu \mathrm{m} \mathrm{CO} 2 \mathrm{DIAL}$ application", Applied Optics, 56, 7577 (2017)

[3] A. Gassenq, L. Milord, J. Aubin, K. Guilloy, S. Tardif, N. Pauc, J. Rothman, A. Chelnokov, J. M. Hartmann, V. Reboud, and V. Calvo, "Gamma bandgap determination in pseudomorphic GeSn layers grown on Ge with up to 15\% Sn content”, Appl. Phys. Lett. 109, 242107 (2016)

[4] Sun, X., Abshire, J.B., Beck, Reiff, K., Yang, G., Optics Expressn 25, 16589 (2017)

[5] D. E. Atkinson, D. N. B. Hall; I. M. Baker; S. B. Goebel; S. M. Jacobson; C. Lockhart; E. A. Warmbier, "Nextgeneration performance of SAPHIRA HgCdTe APDs", Proc. SPIE, 9915, 99150N (2016)

[6] Rothman, J., Pes, S., Bleuet, P., Abergel, J., Gout, S., Nicolas, J.-A., Rostaing, J.-P., Rentet, S., Mathieu, L., Le Perchec, J., "Meso-photonic Detection with HgCdTe APDs at High Count Rates", J. Electron. Mater., 49, 6881 (2020)

[7] J. Rothman, L. Mollard, S. Goût, L. Bonnefond, J. Wlassow, "History-Dependent Impact Ionization Theory Applied to HgCdTe e-APDs", J. Electron. Mater., 40, 1757 (2011) 
[8] J. Rothman, L. Mollard, S. Bosson, G. Vojetta, K. Foubert, S. Gatti, G. Bonnouvrier, F. Salveti, A. Kerlain, O. Pacaud, "Short-Wave Infrared HgCdTe Avalanche Photodiodes", J. Electron. Mater., 41, 2928(2012)

[9] J. Rothman, "Physics and limitations of HgCdTe APDs: a review “, J. Electron. Mater., 35, 1166 (2018)

[10] J. Rothman, K. Foubert, G. Lasfargues, C. Largeron.," Response Time Measurements in Short-Wave Infrared HgCdTe e-APDs” J. Electron. Mater., 43, 2947 (2014) 